\title{
Discussion on Coupling Relationship between the Negative Transfer and Pragmatic Faults in College English Teaching Practice of Translating Chinese into English
}

\author{
Xuejun Lu \\ Dalian Maritime University \\ Dalian, China
}

Keywords: Negative pragmatic transfer; Pluralistic decision-making; Factors associated; Model analysis

\begin{abstract}
Negative pragmatic transfer of mother tongue is the main cause of pragmatic failure in the teaching practice of Chinese to English of Chinese colleges. The analysis and understanding of this cause is the basic requirement of the college English education system. This paper establishes the correlation analysis model of negative transfer in the practice of Chinese to English using decision analysis thought based on the cognition and current situation of negative pragmatic transfer of English in Chinese colleges. It analyzes the dynamic process of students in the practice of Chinese to English. It also analyzes the coupling of negative pragmatic transfer and pragmatic failure of colleges in the teaching practice of Chinese to English based on this situation. This study provides important guidance and practical significance for the development of intercultural communication awareness and ability of foreign language and the abolishment of pragmatic failure.
\end{abstract}

\section{Introduction}

In the teaching process of college English, many teachers and scholars research and discuss the pragmatic failure and negative pragmatic transfer [1]. For example, "analysis of the error of Chinese to English in college English teaching process: Negative Pragmatic Transfer" of Xuejun Lu treats negative pragmatic transfer of mother tongue as the main reason of pragmatic failure in the practice of Chinese to English. He analyzes the main reason and proposes a series of training program to strengthen students' intercultural communicative competence which plays a very important practical significance for the abolishment of pragmatic failure [2,3]. It uses task teaching mode which can continue strengthen and enhance teachers' self-learning ability.

\section{The cognitive status of English negative pragmatic transfer}

In order to survey the attitude of students and teachers of Chinese college in English teaching on English negative transfer, we can treat the 60 college English teachers of different titles in different regions and 900 college English majors and non-English major students of different regions as the research object. We can survey it using the mode of questionnaires and interviews. The survey of teachers contains five questions which are shown in Table 1.

The data of Table 1 involves the following content: survey questions of college English pragmatic negative transfer, negative impact of negative pragmatic transfer, the correction of pragmatic failure, the correction is timely or not, the education of negative transfer knowledge of teachers on students and the understanding of students on English learning negative transfer. The radar profile is shown in Figure 1.

Tab 1. The statistical of open investigation on teachers

\begin{tabular}{|l|l|l|l|l|l|}
\hline The problem teachers related & Agree it very much & Agree & Have no idea & Don't agree & Don’t agree very much \\
\hline $\begin{array}{l}\text { There are only negative effects on } \\
\text { the cross-cultural communication }\end{array}$ & $13.33 \%$ & $50.00 \%$ & $8.33 \%$ & $23.33 \%$ & $5.00 \%$ \\
\hline $\begin{array}{l}\text { Negative pragmatic transfer should } \\
\text { be corrected }\end{array}$ & $10.00 \%$ & $48.33 \%$ & $3.33 \%$ & $30.00 \%$ & $8.33 \%$ \\
\hline
\end{tabular}




\begin{tabular}{|l|l|l|l|l|l|}
\hline $\begin{array}{l}\text { The correction of students' negative } \\
\text { pragmatic transfer must be timely }\end{array}$ & $6.67 \%$ & $40.00 \%$ & $3.33 \%$ & $25.00 \%$ & $8.33 \%$ \\
\hline $\begin{array}{l}\text { We should understand students' } \\
\text { awareness of negative pragmatic } 13.33 \% \\
\text { transfer }\end{array}$ & $36.67 \%$ & $8.33 \%$ & $35.00 \%$ & $15.00 \%$ \\
\hline $\begin{array}{l}\text { The teacher who had learn negative } \\
\text { pragmatic transfer theory }\end{array}$ & $0.00 \%$ & $33.33 \%$ & $0.00 \%$ & $45.00 \%$ & $21.67 \%$ \\
\hline
\end{tabular}

From the Table 1 and Figure 1, we can see that 2/3 teachers believe that the English negative transfer has negative impact on cross-cultural communication which descripts that English negative pragmatic transfer attract the attention of college English teachers. More than $50 \%$ of the teachers considered that the English negative pragmatic transfer need to be corrected. Different regions and teachers have difference opinions on the correction of negative pragmatic transfer. $46.7 \%$ of teachers think that it needs to correct timely to deepen the impression of the students [4-7]. The vast majority of teachers think they have no idea about pragmatic negative transfer theory. The questionnaire of English negative transfer status in different regions is shown in Table 2.

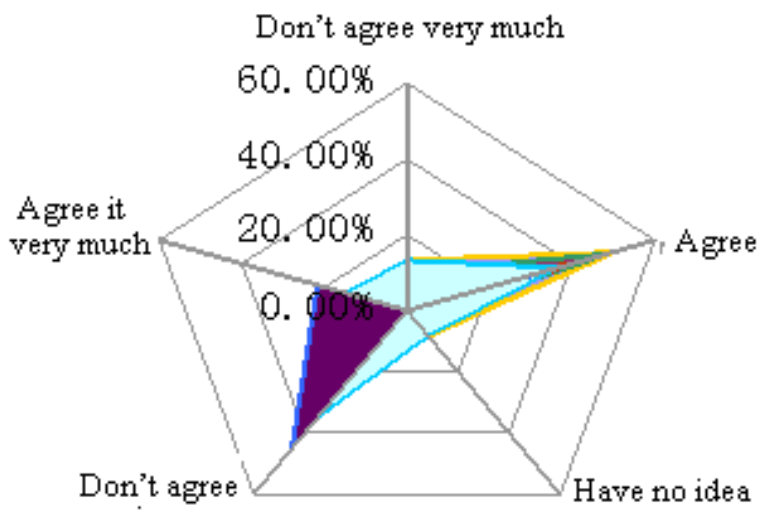

Fig 1. The radar profile of negative transfer attitude in different regions and different titles of college English teachers

Tab 2. The questionnaire of English negative pragmatic transfer status

\begin{tabular}{|l|l|l|l|l|l|}
\hline The problem students related & Agree it very much & Agree & Have no idea & Don't agree & Don't agree very much \\
\hline $\begin{array}{l}\text { There are negative pragmatic } \\
\text { transfers in cross-cultural } 13.33 \% \\
\text { communication }\end{array}$ & $50.00 \%$ & $8.33 \%$ & $23.33 \%$ & $5.00 \%$ \\
\hline $\begin{array}{l}\text { Negative pragmatic transfer should } 10.00 \% \\
\text { be corrected }\end{array}$ & $48.33 \%$ & $3.33 \%$ & $30.00 \%$ & $8.33 \%$ \\
\hline $\begin{array}{l}\text { The correction of negative } \\
\text { pragmatic transfer must be timely }\end{array} 6.67 \%$ & $40.00 \%$ & $3.33 \%$ & $25.00 \%$ & $8.33 \%$ \\
\hline $\begin{array}{l}\text { It is sorry to correct in public } 13.33 \% \\
\text { Frequent correction of negative } \\
\text { pragmatic transfer will lose interest } 0.00 \% \\
\text { in learning }\end{array}$ & $36.67 \%$ & $8.33 \%$ & $35.00 \%$ & $15.00 \%$ \\
\hline
\end{tabular}

As is shown in Table 2, the survey of teachers involves the following five questions: whether they are related to negative pragmatic transfer; if it is need to correct when there are negative pragmatic transfers and if it is timely; the feeling of correction in public place, if he or she will lose the interest in learning because of the frequent pragmatic negative transfer. We can survey the students of five levels and the final statistical results and radar chart is shown in Figure 2. 


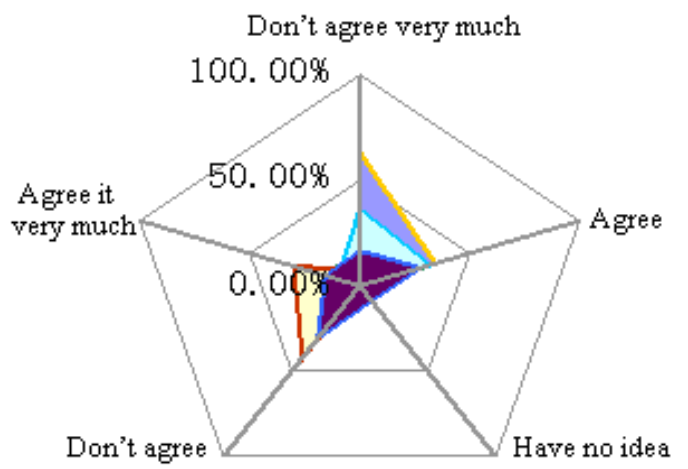

Fig 2. The radar profile of negative transfer attitude in different regions and different titles of college students

From Table 2 and Figure 2, we can see that more than 2/3 of the students consider that there are English Pragmatic negative migration behaviors in actual English learning practice. 1/3 of the students think that there is negative transfer phenomenon in cross-cultural communication $[8,9]$. Nearly half of the students think that it is necessary to correct negative pragmatic transfer when there is negative transfer phenomenon. Half of the students think that there is no need to correct[10]. Nearly $70 \%$ of the students considered that it is not better to correct negative transfer in public that will make the students embarrassing. $44.45 \%$ of students believe that the long-term and frequent English negative pragmatic transfer correct will cause the loss of interest in English learning. 46.67\% believe that he will not lose interest in English learning because of the frequent correct of negative pragmatic transfer.

\section{The analysis of negative transfer process of college English translation teaching practice}

The pragmatic failure of English transfer attracts the attention of vast majority of scholars. The description of English pragmatic negative transfer is different. They have one thing in com mon that when the learners use the second language and the mother tongue, there are unified manifestations of the event. The generation mode of negative pragmatic transfer of Chinese to English is shown in Figure 3.

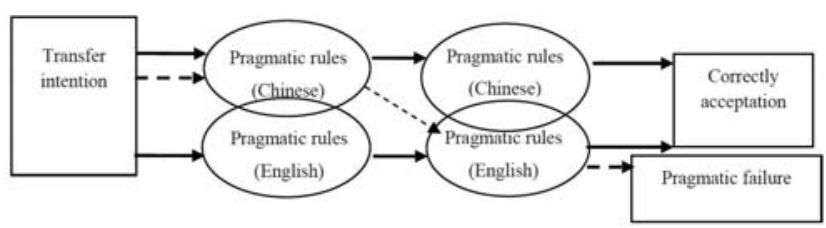

(a)

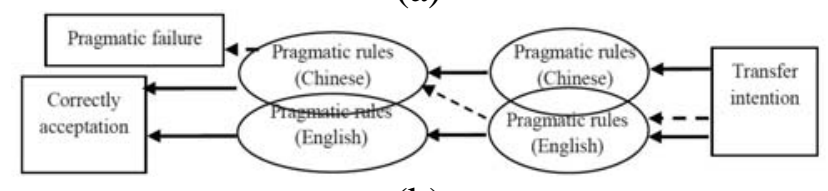

(b)

Fig 3. The figure of negative transfer of Chinese to English

As is shown in Figure 3(a) and Figure 3(b), we can see the whole process of pragmatic transfer of Chinese to English. When pragmatic rules meet the second language in the process of transfer, the entire information will be accepted correctly. When pragmatic rules cannot meet the second language in the process of transfer, there will appear pragmatic failure. In the multivariate analysis of influencing factors of Chinese to English of college English teaching practice, we can define $\hat{\beta}$ as the pragmatic rules and the pragmatic rules of English are the linear unbiased estimation of $\beta$ and $D(\hat{\beta})=\operatorname{Var}(\hat{\beta})=\sigma^{2}\left(X^{T} X\right)^{-1}$. Because $\hat{\beta}=\left(X^{T} X\right)^{-1} X^{T} Y$ is the linear function of $Y$, the pragmatic rules of Chinese are the linear estimation of language rules of English:

$E(\hat{\beta})=\left(X^{T} X\right)^{-1} X^{T} E(Y)=\hat{\beta}=\left(X^{T} X\right)^{-1} X^{T} X \beta=\beta$ 


$$
D(\hat{\beta})=\left(X^{T} X\right)^{-1} X^{T} D(Y) X^{T}\left(X^{T} X\right)^{-1}=\sigma^{2}\left(X^{T} X\right)^{-1}
$$

According to formula (1), formula (2) and Figure 3(a), the pragmatic rules of English and Chinese language are transfer with their own rules. We can use the Chinese rule in the rules of English. If it is not uniform, $E(\vec{e})=O, D(\vec{e})=\sigma^{2}(I-H)$, it will appear pragmatic failure and incorrect acceptation. It is negative pragmatic transfer which means that pragmatic rules $\hat{\beta}$ of Chinese is the Linear Unbiased Estimation of pragmatic rules $\beta$ of English. Because $\left(X^{T} X\right)^{-1}$ is non-diagonal matrix, each component of $\hat{\beta}$ is related.

In Figure 3(b), the second language transfers with the inherent rules. When the English language rules have inconsistency with Pragmatic rules of Chinese, due to $_{\vec{e}=(I-H) Y}, E(\vec{e})=(I-H) E(Y)=(I-H) X \beta=O$. It will produce accept errors and pragmatic failure. It is negative pragmatic transfer:

$$
D(\vec{e})=(I-H) D(Y)(I-H)^{T}=\sigma^{2}(I-H)
$$

This nature shows that the various components of the residual vector of Chinese pragmatic rules are related with each other. The Ming residuals $\vec{e}$ of second language are not related with the least squares estimation of English pragmatic rules $\beta$ and Chinese Pragmatic rules $\hat{\beta}$. In the chart of negative pragmatic transfer of Chinese to English, we should pay attention to the main expression process which treats the Chinese and English Learning as the main object and it does not apply to all negative transfer processes. Negative pragmatic transfer not denies the normalization of language rules of different languages. The occurrence of pragmatic transfer is closely link with the grammar of language rules in a certain extent.

\section{The coupling relationship between pragmatic failure and negative pragmatic transfer in teaching process of Chinese to English}

The key factor of pragmatic failure and negative pragmatic transfer in the teaching practice of Chinese to English is internal language between Chinese and English [12]. It mainly establishes the bridge between Chinese and English. For example, if we want to analyze the coupling relationship of negative pragmatic transfer and pragmatic failure in the practice of Chinese to English, we must know the mechanism of this internal language. Its learning objective is different with mother tongue and English. It is in the middle of English interpretation and Chinese translation in the practice. It promotes the learners to accurate expression in the process of Chinese to English in the learning process. The relationship among the three is shown in Figure 4.

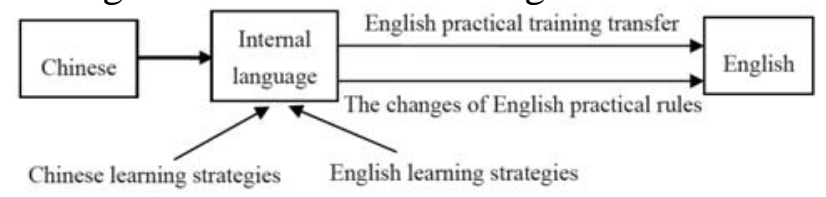

Fig 4. The relationship diagram of Pragmatic Failure and negative pragmatic transfer in English teaching practice of Chinese to English

From the Figure 4, we can see that Chinese to English treats the internal language as intervention and Chinese as the starting point. There are more and close with the gradual deepening of English target translation[11,12]. It will generate avoidable pragmatic failure in the change of training transfer in English practice and pragmatic rules and the appearance of pragmatic negative transfer phenomenon. If there is significant impact of Chinese as the initial amount in the whole translation process on the random variable of the results of English translation relative to the internal language independent variables ${ }^{X_{1}, X_{2}, \ldots, X_{p}}$.The testing hypotheses is:

$H_{0}: \beta_{1}=\beta_{2}=\ldots \beta_{P}=0$

$H_{1}: \beta_{i} \neq 0,1 \leq i \leq p$ 
In formula (4), if ${ }^{H_{0}}$ had be accepted, it means that there has no linear relationship between $y$ and $x_{1}, x_{2}, \ldots, x_{p}$.The internal language rules are change in the practice of Chinese to English. It is inevitable of the appearance of pragmatic failure in the process of Chinese to English. When there are linear relationships between random variables of English translation results and the internal language independent variables $x_{1}, x_{2}, \ldots, x_{p}$, the value of internal language independent variables $x_{1}, x_{2}, \ldots, x_{p}$ will result in the difference of Chinese to English results. At the same time, beside any factors except the linear relationship between the random variable of the English translation results ${ }^{y}$ and internal language independent variables $x_{1}, x_{2}, \ldots, x_{p}$, for example, the non-linear effects of internal language independent variables $x_{1}, x_{2}, \ldots, x_{p}$ on English translation results and the influence of random factors. The result after the Normalization is:

$$
\bar{y}=\frac{1}{n} \sum_{i=1}^{n} y_{i}
$$

According to formula (5), in order to avoid pragmatic failure in intercultural communication in college English learning process, we can get the total sum of squared differences of the data:

$$
\text { SST }=\sum_{i=1}^{n}\left(y_{i}-\bar{y}\right)^{2}
$$

In formula (6), the results of pragmatic failure of Chinese to English delete the linear relationship

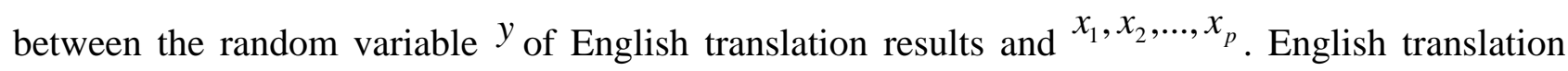
results result from the pragmatic failure of Chinese to English display the fluctuation of $y_{1}, y_{n}, \ldots, y_{p}$. When $\mathrm{SEE}=0$, the error value of English translation can be fitted by the linear relationship precisely. If SSE is larger, the deviation between the error value of English translation and the linear fitted values of English negative pragmatic transfer is larger.

According to negative pragmatic transfer and pragmatic failure in college English translation teaching practice, we can introduce the coupling relationship between them which can improve the attention of teachers and students on the importance of English culture teaching and the importance of cultural differences and avoid pragmatic failure in textbooks. We also should develop students' cross-cultural awareness and interpersonal skill, establish and change the new concept of teaching which makes teaching adapt to the social needs and makes the culture collections penetrate to all aspects of English practice of Chinese to English.

\section{Conclusion}

With the continuous development of China's higher education, it showed a new situation in recent years. In order to adapt to this new situation and the deepening of education reform, we should improve the quality of teaching and meet the needs of English talent of the nation and society in the new era. Modern teaching practice should re-understand the guiding significance of negative pragmatic transfer on college English teaching. We should maintain an objective attitude to look at the coupling between the negative pragmatic transfer and pragmatic failures. We should strengthen the cultivation of students' ability in college English translation teaching practice and accuracy of translation. We also should unify the understanding of original text and grasp the fluency of the translation.

\section{References}

[1] Xunjun Lu. Analysis of the error of Chinese to English in college English teaching process: Negative Pragmatic Transfer [J]. Language and Cultural Studies, 2010 (01):23-24.

[2] Yiping Cui. The pragmatic failure in the perspective of pragmatic negative transfer [J]. Shandong Foreign Language Teaching, 2010 (03):84-86. 
[3] Jie Dong. English negative pragmatic transfer and English teaching [J]. Zhengzhou Railway Vocational and Technical College, 2011 (01):152-157.

[4] Li Tong. The application of pragmatic negative transfer in college English teaching [J]. Xinzhou Teachers University, 2010 (03):121-124.

[5] Zhenbin Guan. Exploration of pragmatic failure in intercultural communication [J]. Huangshi Institute of Technology (Humanities and Social Sciences), 2011 (01):121-123.

[6] Kangzhou Peng. Research on pragmatic failure of cross-cultural communication [J]. Crazy English (Teachers editor), 2011 (01):34-45.

[7] Zhen Zeng. English pragmatic failure and Countermeasures of high school students [D]. Zhangzhou Normal University, 2012 (07):223-224.

[8] Qing Jin.The pragmatic failure of high school students and Revelation of high school teaching [D]. Central China Normal University, 2010(03):132-136.

[9] Lianfen Ou. The analysis of intercultural pragmatic failure and the development of use of pragmatic competence in foreign language teaching [D]. Guangxi normal university, 2010 (02): 203-208.

[10] Shizhu Wang. Research on intercultural Pragmatic failure and the revelation of college English teaching [D]. Chongqing Normal, 2009(04):114-118.

[11] Ziran He. Pragmatics and English learning [M]. Shanghai Foreign Language Education Press, 2010 (03): 32-33.

[12] Kasper, G, Blum-Kulka, S. Inter language Pragmatics: An Introduction [A] He ZhaoXiong. Selected Readings for Pragmatics [C]. Shang hai: Shanghai Foreign Language Education Press, 2010: 715-733. 